\title{
A relação do escore de Lee com a mortalidade pós-operatória em pacientes com fraturas de fêmur proximal*
}

\section{The Relationship between the Lee Score and Postoperative Mortality in Patients with Proximal Femur Fractures}

\author{
Marcelo Teodoro Ezequiel Guerra ${ }^{1}$ Luiz Giglio ${ }^{1}$ João Mauro Mendina Morais ${ }^{1}$ Giovanna Labatut ${ }^{2}$ \\ Monica Cavanus Feijó ${ }^{2}$ Carlos Eduardo Peixoto Kayser ${ }^{2}$

\footnotetext{
${ }^{1}$ Serviço de Ortopedia e Traumatologia, Hospital Universitário de Canoas, Canoas, RS, Brasil

2 Serviço de Ortopedia e Traumatologia, Universidade Luterana do Brasil (Ulbra), Canoas, RS, Brasil
}

\begin{abstract}
Endereço para correspondência Luiz Giglio, MD, Serviço de Ortopedia e Traumatologia, Hospital Universitário de Canoas, Canoas, RS, 92425-020, Brasil (e-mail: Igiglio17@hotmail.com).
\end{abstract}

Rev Bras Ortop 2019;54:387-391.

\section{Resumo \\ Palavras-chave \\ - fraturas do fêmur/ etiologia \\ - fraturas do fêmur/ cirurgia \\ - fraturas do fêmur/ mortalidade \\ - complicações pós- operatórias}

Objetivo Verificar o valor preditivo do escore de Lee para a mortalidade no primeiro ano pós operatório de fraturas de fêmur proximal. O estudo também avaliou a capacidade preditiva isolada de outras variáveis.

Método Uma amostra de 422 pacientes com fraturas do fêmur proximal submetidos a cirurgia foi avaliada neste estudo. Os dados foram coletados por meio de revisão de prontuários, consultas presenciais e contatos telefônicos.

Resultados O escore de Lee foi aplicado em 99,3\% dos pacientes com fraturas de fêmur proximal submetidos a tratamento cirúrgico. A taxa de mortalidade da amostra foi de $22 \%$, a maioria classificada como classe I de risco. O escore de Lee não apresentou associação significativa com a mortalidade $(p=0,515)$. Os valores elevados de creatinina sérica $(p=0,001)$ e a idade $(p=0,000)$ estiveram diretamente associados com o desfecho de morte.

Conclusões O escore de Lee não é preditivo para a mortalidade em um período de um ano após cirurgia de fraturas de fêmur proximal; entretanto, observou-se significância estatística entre a idade e a dosagem sérica da creatinina, isoladamente, com o desfecho de morte.

Objective To verify the predictive value of the Lee score for mortality in a one-year period after proximal femur fracture surgery. The present study also evaluated the isolated predictive capacity of other variables.

* Trabalho desenvolvido no Serviço de Ortopedia e Traumatologia,

Hospital Universitário de Canoas, Canoas, RS, Brasil.

(D) Luiz Giglio's ORCID is https://orcid.org/0000-0002-7144-3170.

recebido

06 de Setembro de 2017

aceito

07 de Dezembro de 2017
DOI https://doi.org/

10.1055/s-0039-1694020. ISSN 0102-3616.
Copyright $\odot 2019$ by Sociedade Brasileira License terms de Ortopedia e Traumatologia. Published by Thieme Revnter Publicações Ltda, Rio de Janeiro, Brazil 


$\begin{array}{ll} & \text { Methods A sample of } 422 \text { patients with surgically-treated proximal femur fractures } \\ & \text { was evaluated. The data were collected through a review of medical records, appoint- } \\ \text { Keywords } & \text { ments, and contact by telephone. } \\ \text { - femoral fractures/ } & \text { Results The Lee score was applied to } 99.3 \% \text { of the patients with proximal femur } \\ \text { etiology } & \text { fractures submitted to surgical treatment. The mortality rate was of } 22 \% \text { of the sample, } \\ \text { - femoral fractures/ } & \text { and the majority were classified as class I risk. The Lee score had no significant } \\ \text { surgery } & \text { association with mortality }(p=0.515) . \text { High levels of serum creatinine }(p=0.001) \text { and } \\ \text { - femoral fractures/ } & \text { age }(p=0.000) \text { were directly associated with death. } \\ \text { mortality } & \text { Conclusion The Lee score wasn't predictive of mortality in a one-year period after } \\ \text { postoperative } & \text { proximal femur fracture surgery; however, a statistical significance was observed } \\ \text { complications } & \text { between age and serum creatinine levels, considered separately, and death. }\end{array}$

\section{Introdução}

As fraturas de fêmur proximal correspondem a uma grande porcentagem das internações por patologias ortopédicas, e estão associadas a taxas elevadas de morbimortalidade. Estima-se que o número de casos pode chegar a 6,26 milhões em 2050, principalmente pela inversão da pirâmide etária populacional a nível mundial. $O$ perfil dos pacientes acometidos é de idade $\geq 70$ anos, brancos, e do sexo feminino em período pós-menopausa. O mecanismo de trauma mais comum é o de baixa energia, relacionado a condições clínicas do paciente, tais como desnutrição, diminuição da acuidade visual e reflexos, uso crônico de medicações, e, principalmente, à progressiva diminuição da densidade mineral óssea. A alta taxa de morbimortalidade e a necessidade de rápida intervenção terapêutica podem dificultar o manejo inicial. Cerca de um terço dos pacientes morre dentro de um ano após o tratamento cirúrgico, e metade permanece com limitações funcionais. Um estudo com 8.930 pacientes relatou uma mortalidade em 30 dias e um ano após a cirurgia de $4 \%$ e $16 \%$, respectivamente. ${ }^{1,2}$ 0 tratamento é predominantemente cirúrgico, e visa à mobilização precoce do paciente. As medidas conservadoras são reservadas para casos específicos, como pacientes sem condições clínicas e/ou não deambuladores. A cirurgia precoce, em menos de 24 horas após o trauma, pode minimizar a chance de complicações secundárias à restrição ao leito, como pneumonia e tromboembolismo venoso. 0 atraso superior a 72 horas no tratamento está associado ao aumento da mortalidade no período entre 30 dias e 1 ano de pós-operatório. ${ }^{3}$ Portanto, a cirurgia em caráter de urgência deve ser preconizada para esses pacientes.

Com vistas a minimizar o hiato entre a admissão hospitalar dos pacientes e a cirurgia, surge também a necessidade de reduzir o tempo despendido na avaliação clínica pré-operatória. A maioria dos pacientes com fraturas do fêmur proximal não pode ser submetida a testes mais específicos de função cardíaca, e, além disso, geralmente apresenta múltiplas comorbidades. Logo, é fundamental a criação de métodos de avaliação pré-operatórios abrangentes, confiáveis e de fácil e rápida aplicação. $O$ índice de risco cardíaco revisado (revised cardiac risk index, RCRI), ou escore de Lee, foi criado este propósito, sendo mundialmente utilizado para estimar o risco de um evento miocárdico peri e pós-operatório imediato. O objetivo deste trabalho foi verificar o valor preditivo do escore de Lee para a mortalidade no período pós-operatório de fraturas de fêmur proximal até um ano. Da mesma forma, se avaliarou a capacidade preditiva isolada de outras variáveis.

\section{Material e Métodos}

Trata-se de um estudo de coorte retrospectivo misto, no qual a população estudada foi composta por pacientes com fraturas da extremidade proximal do fêmur submetidos a cirurgia, com 65 anos ou mais, e internados em um hospital do Sul do Brasil, aos cuidados do Serviço de Ortopedia e Traumatologia, entre junho de 2013 e junho de 2015. O estudo foi aprovado por Comitê de Ética em Pesquisa sob o registro 108356/2016/CAAE 61120016.6.0000.5328.

Foram revisados os prontuários de todos os pacientes com fratura proximal de fêmur no Serviço de Arquivo Médico e Estatístico (SAME) do hospital. A busca foi feita através do código internacional de doenças (CID-10), pelo qual todos os prontuários são classificados. O acompanhamento pós-operatório foi realizado em consultas médicas registradas no sistema interno do hospital, e por contato telefônico com os pacientes e/ou parentes, a fim de minimizar a perda do acompanhamento. Nos casos em que o contato não foi possível, fez-se a verificação no sistema próprio da Secretaria de Saúde do município a fim de rastrear o desfecho. Dessa forma, reduziu-se o número de perdas na amostra.

Os dados foram registrados em uma planilha de Excel 2017 (Microsoft, Redmond, WA, EUA), e compõem um banco para futuros estudos em nosso serviço. A amostra final foi composta de 422 pacientes, e os critérios de exclusão foram óbito pré-operatório e tratamento conservador. As variáveis estudadas foram: idade, sexo, escore de Lee, mecanismo de trauma, mortalidade pós-operatória, lateralidade da fratura, exposição óssea, material de síntese usado, infecção póscirúrgica, lesão neurovascular, trombose venosa periférica, parada cardiorrespiratória, creatinina sérica, comorbidades clínicas, etilismo, tabagismo, hipertensão arterial sistêmica (HAS), diabetes mellitus tipo 2, insuficiência renal, HIV, hepatite $C$, acidente vascular cerebral, insuficiência cardíaca hipertensiva, delirium e cardiopatia isquêmica. 
Para calcular o RCRI, atribui-se um ponto a cada um dos seguintes itens citados: creatinina sérica $>2 \mathrm{mg} / \mathrm{dl}$, histórico de insuficiência cardíaca congestiva, histórico de doença arterial coronariana, histórico de doença cerebrovascular, e histórico de diabetes mellitus insulinodependente. ${ }^{4}$ Adiciona-se um ponto à cirurgia de alto risco. Os procedimentos de quadril são definidos como de risco intermediário. ${ }^{5}$ Portanto, nenhum paciente da amostra foi classificado com o mais elevado estágio de risco. Os desfechos negativos citados foram: infarto agudo do miocárdio, edema pulmonar, fibrilação ventricular, parada cardíaca e bloqueio cardíaco total, e óbito.

Foram usados os testes de associação exata de Fischer e o teste não paramétrico Mann-White para a avaliação estatística. O nível de significância adotado foi de $5 \%(p<0,05)$, e as análises foram feitas no programa Statistical Package for the Social Sciences (SPSS, SPSS, Inc., Chicago, Il, EUA), versão 13.0.

\section{Resultados}

Foram revisados 422 prontuários de junho de 2013 a junho de 2015 , e a amostra final foi composta por $78 \%$ (329) de mulheres e $22 \%$ (93) de homens. Identificamos três grupos etários: 48,1\% (203) com mais de 80 anos, 40,5\% (171) entre 70 e 80 anos, e $11,4 \%$ (48) com menos de 70 anos (- Tabela 1 ). A média de idade foi de 79,8 anos.

O principal mecanismo de trauma foi a queda da própria altura, que correspondeu a 95,7\% (404) dos casos. A topografia das fraturas de fêmur proximal também foi estudada por meio da sua classificação no CID-10: 54.2\% (229) correspondem a fraturas transtrocantéricas, 34,6\% (146), ao colo femoral, e 11,1\% (47), a fraturas subtrocantéricas. A lateralidade encontrada foi de 55\% (232) à esquerda, e de $45 \%$ (190) à direita. É válido mencionar que as fraturas de fêmur proximal com padrões atípicos, como as múltiplas, não foram classificadas nos prontuários eletrônicos, e encontram-se agrupadas na categoria "Outros."

Os implantes mais usados foram parafuso dinâmico de quadril (DHS), em 32\% (135) dos casos, e haste cefalomedular (PFN), em 28,9\% (122). A artoplastia parcial de quadril foi a escolha em $18 \%$ (76) dos casos, e em $13 \%$ (55) optou-se por artroplastia total Os parafuso condilar dinâmico (DCS) e placa de compressão dinâmica (DCP) foram utilizados em 4,3\% (18) e 2,8\% (12) dos casos, respectivamente. Os demais métodos de fixação estão agrupados na categoria "Outros."

Tabela 1 Número total da amostra: gênero e idade

\begin{tabular}{|l|l|l|l|}
\hline Variável & Resposta & Número de casos & $\%$ \\
\hline Gênero & Masculino & 329 & 78,0 \\
\hline & Feminino & 93 & 22,0 \\
\hline Idade & $<70$ anos & 48 & 11,4 \\
\hline & $70-80$ anos & 171 & 40,5 \\
\hline & $>80$ anos & 203 & 48,1 \\
\hline
\end{tabular}

Tabela 2 Total de óbitos e classificação dos pacientes conforme o escore de Lee

\begin{tabular}{|l|l|l|l|}
\hline Variável & Resposta & Número de casos & $\%$ \\
\hline Óbito & Não & 343 & 81,3 \\
\hline & Sim & 79 & 18,7 \\
\hline Escore de Lee & I & 268 & 63,5 \\
\hline & II & 112 & 26,5 \\
\hline & III & 36 & 8,5 \\
\hline & IV & 3 & 0,7 \\
\hline & Outros & 3 & 0,7 \\
\hline
\end{tabular}

A taxa de contaminação encontrada nos prontuários e nas descrições cirúrgicas foi de $0,5 \%$ (2 casos). Trombose venosa profunda (TVP) ocorreu em $0,5 \%$ (2) dos casos, e parada cardiorrespiratória (PCR), em 2,1\% (9) dos casos. Não houve casos de lesão neurovascular.

O escore de Lee foi aplicado em 99,3\% (419) dos pacientes com fraturas de fêmur proximal submetidos a procedimento cirúrgico, 63,5\% (268) dos quais foram classificados como classe I, 26,5\% (112), como classe II, 8,5\% (36), como classe III, e $0,7 \%$ (3), como classe IV. Os pacientes não classificados estão agrupados na categoria "Outros." A taxa de mortalidade da amostra foi de 18,7\% (93 casos) (- Tabela 2 ).

Os dados analisados demonstram que o escore de Lee não apresenta associação significativa com a mortalidade dos pacientes ( $p=0,515)$, uma vez que a maior percentagem de desfechos com morte (60,8\%) foi daqueles classificados como de baixo risco pelo RCRI. Além disso, apenas 1,3\% do total de óbitos pertencia ao grupo de mais alto risco (-Tabela 3 ). Outro achado é que os valores elevados de creatinina sérica $(p=0,001)$ e a idade $(p=0,000)$ estão diretamente associados com o desfecho de morte (-Tabela 4). A ocorrência de óbito foi mais prevalente na faixa etária acima de 80 anos, e correspondeu a $68,8 \%$ da amostra $(p=0,023)$ (-Tabela 5).

\section{Discussão}

Este estudo teve como objetivo avaliar a eficácia do escore de Lee como ferramenta de avaliação pré-cirúrgica para prever a mortalidade no período de até um ano após cirurgia de

Tabela 3 Relação entre a classificação no escore de Lee com a mortalidade

\begin{tabular}{|l|l|l|l|l|l|l|}
\hline Variável & \multirow{2}{*}{ Resposta } & \multicolumn{4}{|l|}{ Óbito } & \multirow{2}{*}{ valor-p } \\
\cline { 3 - 6 } & & \multicolumn{2}{|l|}{ Não } & \multicolumn{2}{l|}{ Sim } & \\
\cline { 3 - 6 } & & $n$ & $\%$ & $n$ & $\%$ & \\
\hline $\begin{array}{l}\text { Escore } \\
\text { de Lee }\end{array}$ & I & 220 & 64,7 & 48 & 60,8 & 0,515 \\
\hline & II & 91 & 26,8 & 21 & 26,6 & \\
\hline & III & 27 & 7,9 & 9 & 11,4 & \\
\hline & IV & 2 & 0,6 & 1 & 1,3 & \\
\hline
\end{tabular}


Tabela 4 Relação entre níveis de creatinina e idade com a mortalidade

\begin{tabular}{|l|l|l|l|l|l|}
\hline Variável & Óbito & $\boldsymbol{n}$ & Média & $\begin{array}{l}\text { Desvio } \\
\text { padrão }\end{array}$ & valor-p \\
\hline Creatinina $>$ & Não & 343 & 0,97 & 0,66 & 0,001 \\
\hline $2 \mathrm{mg} / \mathrm{dL}$ & Sim & 79 & 1,45 & 1,51 & \\
\hline Idade & Não & 343 & 79,08 & 8,12 & 0,000 \\
\hline & Sim & 79 & 83,10 & 7,84 & \\
\hline
\end{tabular}

Tabela 5 Relação entre gênero e faixa etária com as taxas de mortalidade

\begin{tabular}{|l|l|l|l|l|l|l|}
\hline \multirow{2}{*}{ Variável } & \multirow{2}{*}{ Resposta } & \multicolumn{3}{|l|}{ Óbito } & \multirow{2}{*}{ valor-p } \\
\cline { 3 - 6 } & & Não & \multicolumn{2}{l|}{ Sim } & \\
\cline { 3 - 6 } & & $n$ & $\%$ & $n$ & $\%$ & \\
\hline Gênero & Feminino & 271 & 79,0 & 58 & 79,4 & \\
\hline & Masculino & 72 & 21,0 & 21 & 26,6 & \\
\hline Idade & $<70$ anos & 44 & 2,8 & 4 & 5,1 & \\
\hline & $70-80$ anos & 144 & 42,0 & 27 & 34,2 & 0,23 \\
\hline & $>80$ anos & 155 & 45,2 & 48 & 60,8 & \\
\hline
\end{tabular}

fraturas de fêmur proximal, e analisar as variáveis individuais para correlação com o desfecho de morte. Atualmente, existe uma divergência na literatura sobre a qualidade dos métodos avaliativos pré-operatórios simples em diversas áreas cirúrgicas. ${ }^{6}$ Dentre eles, o escore De Lee. Trabalhos prévios demonstram que em procedimentos ortopédicos o escore de Lee apresentou limitação de sua capacidade de prever desfechos desfavoráveis. Na amostra total de 227 pacientes submetidos a cirurgias ortopédicas eletivas, Vetrugno et $\mathrm{al}^{7}$ verificaram que a maioria das complicações pós-operatórias ocorreu nos pacientes classificados com escores de Lee baixo e intermediário (I e II, respectivamente). No mesmo estudo, os autores compararam a eficácia desse escore com a dosagem pré e pós-operatória de peptídeo natriurético do tipo $\mathrm{B}$ (PNB), e concluíram que este marcador apresentou melhor capacidade preditiva para desfechos desfavoráveis. Em outro artigo, Ackland et $\mathrm{al}^{8}$ usaram uma modificação do RCRI ( $\mathrm{mRCRI}$ ) que contava com alterações do eletrocardiograma, hipertensão não controlada, e idade acima de 70 anos, para avaliar a morbimortalidade pósoperatória em uma amostra de 560 pacientes submetidos a artroplastias de joelho e quadril eletivas. Como resultado, houve aumento da especificidade. Um estudo norte-americano feito por Waterman et al, ${ }^{9}$ criou uma nova ferramenta de avaliação pré-operatória de simples aplicação para prever o risco de eventos cardiológicos intra e pós-operatórios em pacientes submetidos a artroplastia total de joelho e quadril. $\mathrm{O}$ chamado total joint arthroplasty cardiac risk index tem apenas três variáveis: hipertensão, idade $\geq 80$ anos, e histórico de doença cardíaca. Na grande amostra, que contava com 85.129 pacientes, evidenciou-se melhor eficácia da predição de desfechos desfavoráveis desse novo escore em compara- ção direta com o de Lee. Estes trabalhos reforçam nosso estudo ao apresentarem a eficácia de fatores isolados na predição de morbimortalidade em cirurgias ortopédicas.

Existem, por outro lado, estudos relacionados a cirurgias não ortopédicas que relatam maior fidedignidade na avaliação pré-operatória com o escore de Lee em relação a outros métodos. Uma pesquisa ${ }^{10}$ que envolveu cirurgia de bypass aórtico evidenciou uma relevância significativa do RCRI com o risco de mortalidade pós-operatória em 30 dias e 1 ano. Porém, esse trabalho não levou em conta fatores como indicação cirúrgica, procedimento de emergência, e idade, que ofereceriam informações adicionais ao prognóstico. ${ }^{10}$ Em seu estudo, Archan et al $^{11}$ consideraram o escore de Lee uma ferramenta útil na estratificação de pacientes de alto risco submetidos a reparo vascular.

Há métodos mais específicos para a avaliação das condições pré-operatórias dos pacientes, apesar da maioria envolver custos elevados e certa dificuldade técnica para realizar no setor de emergência. É sabido que grande parte dos pacientes com fraturas de fêmur proximal que aguardam procedimento cirúrgico não tolera medidas mais objetivas de avaliação cardiorrespiratória. ${ }^{12}$ Segundo Canty et $\mathrm{al}^{13}$ a ecocardiografia transtorácica pré-operatória pode reduzir a mortalidade em pacientes vítimas de fratura de quadril, sem gerar atrasos no procedimento. Porém, há questões inerentes à nossa realidade, uma vez que os recursos são limitados e impõem restrições a métodos de avaliação mais avançados.

A avaliação do escore de Lee pode ser comparada a outra ferramenta de avaliação pré-cirúrgica: o escore da American Society of Anesthesiologists (ASA). Um estudo brasileiro ${ }^{14}$ demonstrou correlação entre este último método e a mortalidade após cirurgia de correção de fraturas do quadril. ${ }^{14}$ Ambos levam em conta critérios que isoladamente têm importância significativa no surgimento de fatalidades. A insuficiência cardíaca, por exemplo, é uma das condições cardíacas com o maior índice de mortalidade em procedimentos não cardiológicos, bem como a estenose aórtica e a hipertensão pulmonar. ${ }^{13}$ O RCRI pode apresentar certa vantagem teórica, por incluir infarto agudo do miocárdio e AVC entre suas variáveis, que foram as principais causas de morte em análise feita recentemente. ${ }^{14}$

A creatinina também exibe relevância no período perioperatório. Já foi demonstrado que acréscimos maiores do que $0,3 \mathrm{mg} / \mathrm{dl}$ no valor basal podem servir como marcador de infecção em pacientes idosos com fratura de quadril. ${ }^{15} \mathrm{Da}$ mesma fora, a idade é um dado já indicado como preditivo de desfecho negativo, demonstrado em outros estudos. ${ }^{16-19}$ Comorbidades mais sérias e em número maior, como osteoporose, aterosclerose, doenças cardíacas, diabetes, e insuficiências cerebral e renal são mais comumente encontradas nos pacientes idosos. 0 presente estudo está em concordância com a literatura, visto que os valores da dosagem sérica de creatinina e a idade, individualmente, apresentaram correlação estatística com a mortalidade. É pertinente a menção de que a amostra deste trabalho conta com um número significativamente alto de pacientes com idade elevada, acima da média estatística de outros trabalhos revisados. 
Contamos com 203 pacientes com idade superior a 80 anos, 0 que corresponde a $48,1 \%$ do total estudado.

Durante a pesquisa da literatura sobre esse tema, evidenciamos que existe uma carência de trabalhos que tratem do desfecho de óbito predito pelo escore de Lee em cirurgias não cardíacas de natureza traumatológica.

O presente estudo gerou um vasto banco de dados que poderá ser usado para a criação de novos trabalhos em nosso serviço, além de instigar a criação de uma nova, mais objetiva e simples ferramenta de avaliação pré-operatória. Acreditamos também ter contribuído para a atenção às condições que oferecem maiores riscos aos pacientes.

\section{Conclusão}

Concluímos que o escore de Lee não é preditivo de mortalidade no período de um ano após cirurgia de fraturas de fêmur proximal. Porém, houve significância estatística entre a idade e dosagem sérica da creatinina, isoladamente, com o desfecho de morte. As demais variáveis não apresentaram associação com os óbitos da amostra.

Conflitos de Interesse

Os autores declaram não haver conflitos de interesse.

\section{Referências}

1 Lauritzen JB, Schwarz P, Lund B, McNair P, Transbøl I. Changing incidence and residual lifetime risk of common osteoporosisrelated fractures. Osteoporos Int 1993;3(03):127-132

2 Lawrence VA, Hilsenbeck SG, Noveck H, Poses RM, Carson JL. Medical complications and outcomes after hip fracture repair. Arch Intern Med 2002;162(18):2053-2057

3 Shiga T, Wajima Z, Ohe Y. Is operative delay associated with increased mortality of hip fracture patients? Systematic review, meta-analysis, and meta-regression. Can J Anaesth 2008;55(03): 146-154

4 Lee TH, Marcantonio ER, Mangione CM, et al. Derivation and prospective validation of a simple index for prediction of cardiac risk of major noncardiac surgery. Circulation 1999;100(10): 1043-1049

5 Fleisher LA, Beckman JA, Brown KA, et al; American College of Cardiology; American Heart Association Task Force on Practice Guidelines (writing Committee to Revise the 2002 Guidelines on Perioperative Cardiovascular Evaluation for Noncardiac Surgery); American Society of Echocardiography; American Society of Nuclear Cardiology; Heart Rhythm Society; Society of Cardiovascular Anesthesiologists; Society for Cardiovascular Angiography and Interventions; Society for Vascular Medicine and Biology; Society for Vascular Surgery. ACC/AHA 2007 guidelines on perioperative cardiovascular evaluation and care for noncardiac surgery: a report of the American College of Cardiology/American Heart Association Task Force on Practice Guidelines (Writing Committee to Revise the 2002 Guidelines on Perioperative
Cardiovascular Evaluation for Noncardiac Surgery) developed in collaboration with the American Society of Echocardiography, American Society of Nuclear Cardiology, Heart Rhythm Society, Society of Cardiovascular Anesthesiologists, Society for Cardiovascular Angiography and Interventions, Society for Vascular Medicine and Biology, and Society for Vascular Surgery. J Am Coll Cardiol 2007;50(17):e159-e241

6 Barnett S, Moonesinghe SR. Clinical risk scores to guide perioperative management. Postgrad Med J 2011;87(1030):535-541

7 Vetrugno L, Langiano N, Gisonni R, et al. Prediction of early postoperative major cardiac events after elective orthopedic surgery: the role of B-type natriuretic peptide, the revised cardiac risk index, and ASA class. BMC Anesthesiol 2014;14:20

8 Ackland GL, Harris S, Ziabari Y, Grocott M, Mythen M; SOuRCe Investigators. Revised cardiac risk index and postoperative morbidity after elective orthopaedic surgery: a prospective cohort study. Br J Anaesth 2010;105(06):744-752

9 Waterman BR, Belmont PJ Jr, Bader JO, Schoenfeld AJ. The Total Joint Arthroplasty Cardiac Risk Index for Predicting Perioperative Myocardial Infarction and Cardiac Arrest After Primary Total Knee and Hip Arthroplasty. J Arthroplasty 2016;31(06):1170-1174

10 Moitra VK, Flynn BC, Mazzeffi M, Bodian C, Bronheim D, Ellis JE. Indication for surgery, the revised cardiac risk index, and 1-year mortality. Ann Vasc Surg 2011;25(07):902-908

11 Archan S, Roscher CR, Fairman RM, Fleisher LA. Revised Cardiac Risk Index (Lee) and perioperative cardiac events as predictors of long-term mortality in patients undergoing endovascular abdominal aortic aneurysm repair. J Cardiothorac Vasc Anesth 2010;24 (01):84-90

12 Older P, Hall A, Hader R. Cardiopulmonary exercise testing as a screening test for perioperative management of major surgery in the elderly. Chest 1999;116(02):355-362

13 Canty DJ, Royse CF, Kilpatrick D, Bowyer A, Royse AG. The impact on cardiac diagnosis and mortality of focused transthoracic echocardiography in hip fracture surgery patients with increased risk of cardiac disease: a retrospective cohort study. Anaesthesia 2012;67(11):1202-1209

14 Guerra MT, Thober TA, Bigolin AV, de Souza MP, Echeveste S. Hip fracture: Post-operative evaluation of clinical and functional outcomes. Rev Bras Ortop 2015;45(06):577-582

15 Sosa NJ, Laguarda JM, Garcia A, Riba PJ, Duaso E, Bausili JM. Creatinine increase as a marker of infection in hip fracture patients: A historical cohort: 18AP2-3. Eur J Anaesthesiol 2013; 30:246-247

16 Yee DK, Fang C, Lau TW, Pun T, Wong TM, Leung F. Seasonal Variation in Hip Fracture Mortality. Geriatr Orthop Surg Rehabil 2017;8(01):49-53

17 Stott-Eveneshen S, Sims-Gould J, McAllister MM, et al. Reflections on Hip Fracture Recovery From Older Adults Enrolled in a Clinical Trial. Gerontol Geriatr Med 2017;3:2333721417697663. Doi: $10.1177 / 2333721417697663$

18 Guerra MT, Viana RD, Feil L, Feron ET, Maboni J, Vargas AS. Oneyear mortality of elderly patients with hip fracture surgically treated at a hospital in Southern Brazil. Rev Bras Ortop 2016;52 (01):17-23

19 Dedovic Z, Talic-Tanovic A, Resic H, Vavra-Hadziahmetovic N. Mortality among third age patients with hip fracture and high cardiac risk. Med Arh 2013;67(01):42-44 